\title{
Rotational energy surfaces of molecules exhibiting internal rotation
}

Cite as: J. Chem. Phys. 101, 2710 (1994); https://doi.org/10.1063/1.467652

Submitted: 14 March 1994 . Accepted: 28 April 1994 . Published Online: 31 August 1998

Juan Ortigoso, and Jon T. Hougen

\section{ARTICLES YOU MAY BE INTERESTED IN}

Rho-axis-method Hamiltonian for molecules having one methyl rotor and $C_{1}$ pointgroup symmetry at equilibrium

The Journal of Chemical Physics 119, 5505 (2003); https://doi.org/10.1063/1.1599354

The K-rotational labeling problem for eigenvectors from internal rotor calculations: Application to energy levels of acetaldehyde below the barrier

The Journal of Chemical Physics 110, 11688 (1999); https://doi.org/10.1063/1.479115

Rotational energy surfaces and high-J eigenvalue structure of polyatomic molecules The Journal of Chemical Physics 80, 4241 (1984); https://doi.org/10.1063/1.447255
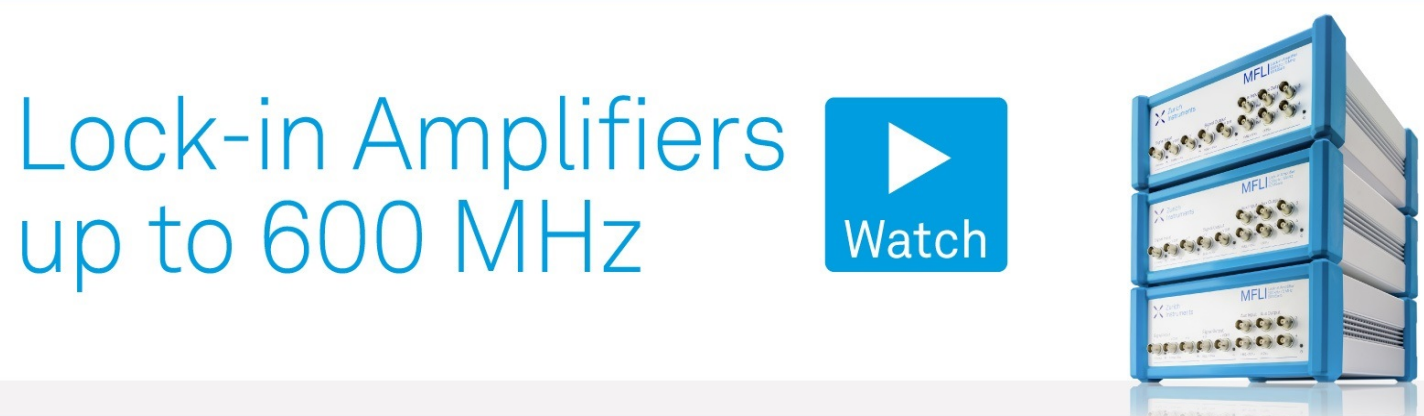

J. Chem. Phys. 101, 2710 (1994); https://doi.org/10.1063/1.467652

101,2710

(c) 1994 American Institute of Physics. 


\title{
Rotational energy surfaces of molecules exhibiting internal rotation
}

\author{
Juan Ortigoso a) and Jon T. Hougen \\ Molecular Physics Division, National Institute of Standards and Technology, Gaithersburg, Maryland 20899
}

(Received 14 March 1994; accepted 28 April 1994)

\begin{abstract}
Rotational energy surfaces [W. G. Harter and C. W. Patterson, J. Chem. Phys. 80, 4241 (1984)] for a molecule with internal rotation are constructed. The study is limited to torsional states at or below the top of the barrier to internal rotation, where the extra (torsional) degree of freedom can be eliminated by expanding eigenvalues of the torsion $-K$-rotation Hamiltonian as a Fourier series in the rotational degree of freedom. For acetaldehyde, considered as an example, this corresponds to considering $v_{t}=0,1$, and 2 (below the barrier) and $v_{t}=3$ (just above the barrier). The rotational energy surfaces are characterized by locating their stationary points (maxima, minima, and saddles) and separatrices. Rather complicated catastrophe histories describing the creation and annihilation of pairs of stationary points as a function of $J$ are found at moderate $J$ for given torsional quantum number $\left(v_{t}\right)$ and symmetry species $(A, E)$. Trajectories on the rotational energy surface which quantize the action are examined, and changes from rotational to vibrational trajectories caused by changes in the separatrix structure are found as a function of $J$ for $v_{t}=2$. The concept of a "best" quantization axis for the molecule-fixed component of the total angular momentum is examined from a classical point of view, and it is shown that labeling ambiguities encountered in the literature for torsion-rotation energy levels, calculated numerically in the rho-axis system, can be eliminated by reprojecting basis-set $K$ values onto an axis passing through an appropriate stationary point on the rotational energy surface.
\end{abstract}

\section{INTRODUCTION}

The concept of a rotational energy surface (RES) was introduced by Harter and Patterson, ${ }^{1}$ and graphically elaborated by Harter. ${ }^{2}$ A RES is a radial plot of the energy of the molecule as a function of the direction of the total angular momentum in the molecule-fixed frame. Harter and Patterson proposed studying rotational motions by considering the trajectory of the tip of the angular momentum vector on the rotational energy surface much as one studies vibrational motions by considering the trajectory of the mass point on the potential energy surface. References 1 and 2 deal with rotational clustering phenomena în spherical tops deformed by quartic centrifugal distortion terms, and in asymmetric tops, both rigid and centrifugally deformed, using RESs based on Hamiltonians expressed as power series expansions of the angular momentum operators.

A RES based on a rigid-bender Hamiltonian for the study of triatomic molecules with a large amplitude bending vibration was proposed by Makarewicz. ${ }^{3}$ The advantage of this treatment is that it avoids the use of power series, which for molecules with large amplitude motions are often divergent or at least slowly convergent.

A new physical phenomenon in $A B_{2}$ molecules, namely. the existence of fourfold clusters caused by strong centrifugal effects, was predicted. ${ }^{4}$ These clusters were interpreted in Ref. 3 using the concept of rotational energy surfaces. This phenomenon has already been verified experimentally for the $\mathrm{H}_{2} \mathrm{Se}$ molecule. ${ }^{5}$

A classification of qualitative changes taking place in RESs of isolated effective rotational Hamiltonians when the

a) Postdoctoral fellow of the Cônsejo Superior de Investigaciones Científicas of Spain 1993-1994. total angular momentum varies has been done. ${ }^{6}$ This work employed the tools of catastrophe theory ${ }^{7}$ to reduce the number of critical changes to a few cases. Very recently, an analysis of the changes suffered by rovibrational states as a consequence of rotational or vibrational excitation has been published. ${ }^{8}$ Other kinds of phenomena such as, e.g., redistribution of energy levels between different branches of the eigenvalue spectrum have been studied ${ }^{9}$ with the use of multiple rotational energy surfaces. This redistribution is related to the existence of degeneracies or conical intersections among different effective rotational energy surfaces.

Other constructions meant to understand semiclassical dynamics have been proposed. Kellman et al. in a series of papers ${ }^{10}$ have introduced the polyad phase sphere to treat coupled vibrations. They obtain a classical phase space from a spectroscopic Hamiltonian using the Heisenberg correspondence principle. This Hamiltonian turns out to be integrable. Catastrophe theory and semiclassical dynamics have been used by Kellman and Xiao to design a method to assign quantum numbers to energy levels of molecules with Fermi resonance. ${ }^{11}$

In the present work, we discuss some aspects of the application of RESs to an asymmetric top containing a hindered symmetric-top internal rotor. Such a system corresponds classically to an asymmetric top containing a gyroscope and can be described by using three Euler angles for the rotation together with an extra angle to describe the internal rotation of the gyroscope. The RES concept is based on the adiabatic separation of the rotation from all other degrees of freedom in the molecule. The usefulness of this approach for internal plus overall rotation problems will depend on the degree of mixing between torsion and rotation. For levels below the top of the barrier to internal rotation, the separation is fairly good (extremely good for a simplified 
model Hamiltonian which neglects centrifugal effects), so that the torsion-rotation problem can be reduced to an effective rotational problem for each torsional state. For levels above the barrier, where the mixing is stronger, several surfaces must be considered at the same time.

As a concrete example in this study,: we consider the acetaldehyde molecule $\mathrm{CH}_{3} \mathrm{CHO}$, focusing on the torsional states below the barrier to internal rotation $\left(v_{t}=0,1\right.$, and 2) and on the first state above the barrier $\left(v_{t}=3\right)$. We present rotational energy surfaces constructed from a model Hamiltonian based on the spectroscopic Hamiltonian obtained from a fit of infrared and microwave transitions of acetaldehyde, ${ }^{12}$ and use these surfaces to determine the best choice of axis for quantizing the projection of the total angular momentum.

\section{RES CONCEPTS}

Some concepts of classical mechanics from the RES literature which are important for the present paper can be summarized as follows: (i) Consider first a body experiencing no external forces and rotating with constant angular momentum $\mathbf{J}$, where $\mathbf{J}$ has both constant magnitude $J$ and constant direction in the laboratory-fixed coordinate system $X, Y, Z$. (ii) The total energy of this body depends on the magnitude of the angular momentum vector $\mathbf{J}$ and on its direction in the body-fixed axis system $x, y, z$; e.g., for rotation about one of the three principal axes of a rigid asymmetric rotor with rotational constants $A, B$, and $C$, this energy would be $A J^{2}, B J^{2}$, or $C J^{2}$ respectively. (iii) By using the direction cosine matrix and Eulerian angle definition of Wilson, Decius, and Cross, ${ }^{13}$ and by placing the laboratoryfixed $Z$ axis along the total angular momentum direction so that $J_{Z}=J$ and $J_{X}=J_{Y}=0$, we find that the components of $\mathbf{J}$ in the molecule-fixed axis system can be written in terms of the magnitude $J$ of the total angular momentum and two of the Eulerian angles in the form

$J_{x}=-J \sin \theta \cos \chi, \quad J_{y}=J \sin \theta \sin \chi, \quad J_{z}=J \cos \theta$.

(iv) Alternatively, the components of $\mathbf{J}$ can be written in terms of the three Eulerian angles $\chi, \theta$, and $\phi$ and their conjugate momenta in the form ${ }^{13}$

$$
\begin{aligned}
& J_{x}=\cot \theta \cos \chi p_{\chi}+\sin \chi p_{\theta}-\csc \theta \cos \chi p_{\phi}, \\
& J_{y}=-\cot \theta \sin \chi p_{\chi}+\cos \chi p_{\theta}+\csc \theta \sin \chi p_{\phi}, \\
& J_{z}=p_{\chi} .
\end{aligned}
$$

(v) The classical rotational motion of a body described by any Hamiltonian $H\left(J_{x}, J_{y}, J_{z}\right)$ written in terms of the angular momentum components of Eqs. (2) can be determined from Hamilton's canonical equations. (vi) The RES is now constructed by plotting in spherical polar coordinates the radius vector $r(\theta, \chi)=H\left(J_{x}, J_{y}, J_{z}\right)=H(\theta, \chi)$ as a function of two Eulerian angles $\theta, \chi$, where the function $H(\theta, \chi)$ is obtained from $J_{x}(\theta, \chi)$, etc. in Eqs. (1). (vii) At this point, introduce the fact that the body not only has constant $\mathbf{J}$, but also constant energy $E$. Then, regardless of the detailed nature of the trajectory for the tip of $\mathbf{J}$ defined by the solution to Hamilton's equations, this trajectory must correspond to a curve on the RES defined by the equation $H(\theta, \chi)=E$. (viii) The trajec- tory defined by the intersection of the constant energy sphere and the RES will be a single point if the intersection takes place at a stationary point of the RES, i.e., at a maximum, minimum, or saddle point. (For a rigid asymmetric rotor with $A>B>C$, maxima, minima, and saddle points of the RES lie on the $A, C$, and $B$ axes, respectively.) Rotational motion for trajectories consisting of a single point is special in the sense that the orientation of the total angular momentum vector in the body-fixed axis system never changes.

\section{MODEL HAMILTONIAN FOR INTERNAL ROTOR MOLECULES}

We specialize consideration in this work to bodies consisting of a molecule containing one symmetric-top internal rotor of $C_{3 v}$ symmetry, i.e., to molecules with one $-\mathrm{CH}_{3}$ top. It is well known that a principal-axis-method Hamiltonian $H_{\text {PAM }}$ for such molecules can be written to a first approximation in the form?

$$
\begin{aligned}
H_{\mathrm{PAM}}= & F\left(p_{\alpha}-\boldsymbol{\rho} \cdot \mathbf{J}\right)^{2}+(1 / 2) V_{3}(1-\cos 3 \alpha)+A^{\prime} J_{z}^{2}+B^{\prime} J_{x}^{2} \\
& +C^{\prime} J_{y}^{2},
\end{aligned}
$$

where $F$ is a rotational constant for the internal rotation motion; $\alpha$ is the angle describing internal rotation; $\boldsymbol{\rho}$ is a vector related to the direction of the axis of internal rotation in the principal axis system; $\mathbf{J}$ is the total angular momentum with components $J_{x}, J_{y}$, and $J_{z}$ in the principal axis system; $V_{3}$ is the barrier height; and $A^{\prime}, B^{\prime}$, and $C^{\prime}$ are the usual asymmetric rotor rotational constants, inversely proportional to the three principal moments of inertia.

We now further specialize to molecules with a plane of symmetry in the equilibrium configuration (e.g., $\mathrm{CH}_{3} \mathrm{OH}$, $\mathrm{CH}_{3} \mathrm{CHO}$, etc). If the plane of symmetry is taken to be the $x z$ plane, then $\rho_{y}=0$ by symmetry. In this case, a rotation about the $y$ axis can be used to eliminate the coupling term $\rho_{x} J_{x}$, yielding a rho-axis-method Hamiltonian ${ }^{15} H_{\text {RAM }}$

$$
\begin{aligned}
H_{\mathrm{RAM}}= & F\left(p_{\alpha}-\rho J_{z}\right)^{2}+(1 / 2) V_{3}(1-\cos 3 \alpha)+A J_{z}^{2}+B J_{x}^{2} \\
& +C J_{y}^{2}+D_{x z}\left(J_{x} J_{z}+J_{z} J_{x}\right),
\end{aligned}
$$

which is often used for numerical computations because the diagonalization of $H_{\mathrm{RAM}}$ can be carried out in two steps. The first step involves diagonalization of only the torsion plus $K$ rotation part of the Hamiltonian. In this first step, only rotational matrix elements with $\Delta K=0$ appear. ${ }^{15}$

Consider now the problem of building a RES from the Hamiltonian $H_{\text {RAM }}$. A rotational energy surface is a classical object based on the adiabatic separation of rotation from all other internal degrees of freedom of the molecule. Therefore it is necessary to eliminate the fast degree of freedom in Eq. (4), i.e., the internal rotation. Perturbation methods can be used to achieve this goal. It is possible, e.g., to eliminate the torsional degree of freedom in the quantum Hamiltonian by means of one or more Van Vleck transformations. The resulting effective rotational Hamiltonian (one for each torsional state) can be written ${ }^{14}$ as a rotational operator plus a series in powers of $\left(\rho J_{z}\right)$. The classical limit of this effective Hamiltonian is immediately obtained upon replacing the angular momentum operators by the expressions given in Eqs. (1). 
An alternative method is to apply classical perturbation theory to the classical Hamiltonian (obtained by substituting coordinates and momenta by their classical analogs). This method has been used, e.g., by Patterson ${ }^{16}$ to generate adiabatic vibrational surfaces for a triply degenerate anharmonic oscillator using first order classical perturbation theory. Perturbation methods, quantum or classical, usually are slowly convergent or divergent. However, as Patterson pointed out, one can use an approximate energy surface, obtained by low order perturbation theory, in connection with some other method, such as adiabatic switching ${ }^{17}$ to calculate quantized trajectories of the system. The only requirement is that the approximate surface must have the same topology as the real surface. ${ }^{16}$ However, it is difficult to know a priori what an adequate topology of the approximate surface is.

A nonperturbative procedure often employed in the literature to build effective rotational energy surfaces for rovibrational problems is to take the classical limit for the components of the angular momentum, but to treat the vibrations quantum mechanically. In our case, this is equivalent to solving a purely torsional problem represented by $H\left(\alpha, p_{\alpha} ; J_{x}, J_{y}, J_{z}\right)$ for fixed Eulerian angles $\theta$ and $\chi$ [see Eqs. (1)] at a number of Eulerian angle pairs sufficient to cover the two sphere. This produces a set of numerical rotational energy surfaces (one surface for each basis function employed in the diagonalization procedure). The surfaces depend on a torsional quantum number (or counting index) $v_{t}$, on the value of the total angular momentum $J$ and on the two Eulerian angles. These surfaces can be fit to appropriate functions of $\theta$ and $\chi$ as, for instance, spherical harmonics.

It is well known ${ }^{14}$ that the torsion plus $K$ rotation energy levels of $H_{\mathrm{RAM}}$ can be fit, in the high barrier limit, to a Fourier series in $\cos [(2 n \pi / 3)(\rho K-\sigma)]$, where $\sigma=0$ or \pm 1 for energy levels of symmetry species $A$ or $E$, respectively, in the molecular symmetry group $G_{6}=C_{3 v}$. We can thus write a rotational energy surface for every torsional state in the following way:

$$
\begin{aligned}
E\left(v_{t}, J ; \theta, \chi\right)= & \sum_{n} F a_{n}^{\left(v_{t}\right)} \cos [(2 n \pi / 3)(\rho J \cos \theta-\sigma)] \\
& +A J^{2} \cos ^{2} \theta+B J^{2} \sin ^{2} \theta \cos ^{2} \chi \\
& +C J^{2} \sin ^{2} \theta \sin ^{2} \chi \\
& -2 D_{x z} J^{2} \sin \theta \cos \theta \cos \chi
\end{aligned}
$$

This Fourier expansion works well until the free rotor limit is reached. In that limiting case, the torsion-rotation energy levels $E_{\text {tr }}$ are given to a first approximation by a different expression

$$
E_{\mathrm{tr}}=F(m-\rho K)^{2}+E_{\text {asym-rotor }},
$$

where $F$ is the rotational constant for internal rotation found in Eqs. (3) and (4), and both $m$ and $K$ are signed integers.

The RES treatment for torsional levels below the barrier can be carried out using one-surface techniques described in the literature, because (i) states of different $A, E$ symmetry (different $\sigma$ ) cannot interact with each other in the absence of hyperfine effects; and (ii) $\Delta \sigma=0, \Delta v_{t} \neq 0$ torsional energy intervals below the barrier top are normally much larger than the Coriolis interaction energies $B J, C J$, and $A K$ for $J$ and $K$ values in ranges normally encountered in room temperature (or lower) experiments. Only the states below the barrier and the first state above the barrier are considered in this paper.

Consider now the RES treatment for torsional levels above the barrier. In particular, can such levels be treated using one-surface techniques, or will they require a multiplesurface treatment? If nondiagonal matrix elements due to the barrier and asymmetric rotor effects are neglected, we find from Eq. (6) that surfaces with different $m$ but the same $J$ can intersect for some values of $\theta$. These touching points have been termed diabolic points ${ }^{9}$ and they are associated with energy level redistribution in the eigenvalue spectrum under the variation of a parameter of the Hamiltonian operator. Such intersections occur when

$$
F\left(m_{1}-\rho J \cos \theta\right)^{2}=F\left(m_{2}-\rho J \cos \theta\right)^{2}
$$

i.e., when

$$
\theta=\arccos \left[\left(m_{1}+m_{2}\right) /(2 J \rho)\right]
$$

where $m_{1}, m_{2}$, and $K$ must all be considered to be signed integers. Since we are interested only in intersections of surfaces of the same symmetry, we must also require in Eqs. (7) and (8) that $m_{1}=m_{2} \bmod 3$.

If off-diagonal matrix elements due to the barrier are included, the intersections given in Eq. (7) will in general become avoided crossings. In principle, this avoided crossing problem is a two-parameter problem, since Hamiltonian matrix elements depend on the two angles $\theta$ and $\chi$. It is well known that such problems give rise to conical intersections between surfaces of the same symmetry, which occur for surfaces $E_{i}$ and $E_{j}$ at points $\theta_{c}, \chi_{c}$ such that the two equations $H_{i i}\left(\theta_{c}, \chi_{c}\right)=H_{j j}\left(\theta_{c}, \chi_{c}\right)$ and $H_{i j}\left(\theta_{c}, \chi_{c}\right)=0$ are both satisfied. The second of these conditions essentially means for the present problem that centrifugal distortion effects must completely annihilate the barrier to internal rotation, so that, in practice, conical intersections will be confined to regions of very high $J$. (For our model Hamiltonian, they will never occur, since centrifugal distortion effects on the barrier height are not considered). For normally accessible $J$ values, we expect only avoided crossings to occur. When the energy gap associated with such an avoided crossing is large with respect to some suitable measure, we expect a onesurface treatment to be adequate; when the gap is small with respect to that measure, we expect that a multiple-surface treatment will be necessary.

\section{RES ANALYSIS OF THE TORSIONAL STATES $V_{T}=0,1,2$, AND 3 OF $\mathrm{CH}_{3} \mathrm{CHO}$}

\section{A. Model Hamiltonian for acetaldehyde}

We first use the method of Sec. III to construct single rotational energy surfaces for the $v_{t}=0,1,2$, and 3 torsional states of acetaldehyde. The quantum Hamiltonian is given in Eq. (4) and the constants have been taken from Ref. 12. The Hamiltonian used there by Kleiner et al. is much more complicated than ours. It involves 48 phenomenological parameters needed to fit microwave and far infrared spectra of acetaldehyde to the experimental accuracy. Many of these 
TABLE I. Parameters" of the model torsion-rotation Hamiltonian used in this work from Ref. 12 .

\begin{tabular}{lrlr}
\hline$A$ & $1.8848741(9)$ & $F$ & $7.6559(6)$ \\
$B$ & $0.3487163(5)$ & $\rho$ & $0.3291(2)$ \\
$C$ & $0.3031777(1)$ & $V_{3}$ & $407.947(2)$ \\
$D_{a b}$ & $-0.122709(3)$ & $V_{6}$ & $-12.918(8)$ \\
\hline
\end{tabular}

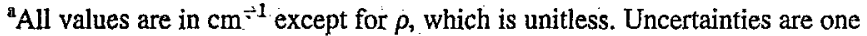
standard deviation in the last digit.

parameters take into account the variation of the barrier with $J(J+1)$ and $K^{2}$, centrifugal distortion effects, etc., and are not directly relevant to the purpose of the present work. We thus set most of the 48 parameters to zero, retaining only $F$, $\rho, V_{3}, V_{6}, A, B, C$, and $D_{a b}$, which are given in Table $\mathrm{I}$. The constant $V_{6}$, which is not in Eq. (4), multiplies the operator $(1 / 2)(1-\cos 6 \alpha)$; its main effect is to modify the shape of the potential barrier.

After taking the classical limit for the rotational operators, a matrix representation for $H_{\mathrm{RAM}}$ is constructed for different values of $\theta$ and $\chi$, using a basis set of 35 free rotor functions. Since the rotational operators have been converted to functions of $\theta$ and $\chi$, they contribute only to the diagonal matrix elements and consequently can be taken out of the matrix. Thus, it is necessary to diagonalize only the torsion plus $K$-rotation part of $H_{\mathrm{RAM}}$. This Hamiltonian matrix is $\chi$ independent. It was diagonalized for $\theta$ values such that $J \cos \theta(=K)$ was an integer with an absolute value less than or equal to 25 .

The resulting eigenvalues were fit to a cosine Fourier series in $(2 n \pi / 3)(\rho K-\sigma)$ as in Eq. (5). The standard deviation of the fits was $2.2 \times 10^{-8}$ for $v_{t}=0,1.4 \times 10^{-7}$ for $v_{t}=1,4.2 \times 10^{-6}$ for $v_{t}=2$, and 0.0089 for $v_{t}=3$, in units of $\mathrm{cm}^{-1}$. The values of the coefficients $F a_{n}$ obtained in the fits are given in Table II. Only three terms were needed to fit the $v_{t}=0$ state, but 15 terms were used in the fit of $v_{t}=3$.

$A$-symmetry RESs cannot interact 'with $E$-symmetry RESs and they can be considered as independent objects. We could thus have fit $A$ and $E$ eigenvalues independently. However, we fit $A$ and $E$ levels together to check the goodness of the Fourier series expansions.

\section{B. Stationary points of the RES}

The rotational energy surface is organized completely by the number and location of stationary points, i.e., those points for which the gradient of the energy with respect to the two spherical coordinates $\theta$ and $\chi$ is zero. Classically, stable trajectories can occur around axes passing through the center of the RES and maxima or minima on the RES. Stable trajectories can also enclose saddle points on the RES, although in that case, they have to also enclose other stationary points because the Poincare index of a closed phase curve ${ }^{18}$ has to be 1 (saddle points have index -1 , maxima and minima +1 , and regular points have index zero).

Stationary points for the model Hamiltonian of acetaldehyde corresponding to the parameters given in Table I were determined for $v_{t}=0,1,2$, and 3 torsional states, for $A$ and $E$ symmetry species, and for various values of $J<35$. The torsional state $v_{t}=3$ of acetaldehyde is the first torsional state above the barrier and distinctive characteristics are expected to occur in its RES. It can be seen that $A$-state $(\sigma=0)$ RESs for this model Hamiltonian are invariant under the $C_{2 h}$ coordinate transformations given in Table III. Similarly, $E$-state $(\sigma= \pm 1)$ RESs are invariant under the $C_{s}$ transformations in Table III. Stationary points for all $A$ and $E$ RESs must therefore be distributed so as to preserve these point group symmetries. (Their distribution will in general, however, preserve no higher point group symmetries.) Furthermore, although we refer to integral values of $J$ in the text, numerical calculations of stationary points, trajectories, etc., were carried out after replacing $J$ in Eq. (5) by its "quantummechanical" value $[J(J+1)]^{1 / 2}$. [It is of interest to note, as the referee points out, that on the one hand, time reversal symmetry is lost in going from the quantum mechanical Hamiltonian in Eq. (4) to the classical RES for $\sigma \neq 0$ in Eq.

TABLE II. Coefficients (in $\left.\mathrm{cm}^{-1}\right)^{\mathrm{n}}$ of the Fourier series fits [as in Eq. (5)] of the eigenvalues of the forsion$K$-rotation Hamiltonian. The standard deviation $(\sigma)$ in $\mathrm{cm}^{-1}$ of the fits is also given.

\begin{tabular}{|c|c|c|c|c|}
\hline & $v_{t}=0$ & $v_{t}=1$ & $v_{t}=2$ & $v_{t}=3$ \\
\hline$F a_{0}$ & $75.355324 .109(3)$ & $218.34829806(2)$ & $341.0134296(7)$ & $443.305(1)$ \\
\hline$F a_{i}^{-}$ & $-0.048729944(4)$ & $1.20965798(2)$ & $-10.4900044(8)$ & $33.601(2)$ \\
\hline $\mathrm{Fa}_{2}$ & $0.000031067(4)$ & $0.01488103(2)$ & $0.8969437(8)$ & $4.533(2)$ \\
\hline $\mathrm{Fa}_{3}$ & $\ldots$ & $0.00030879(2)$ & $-0.1489023(8)$ & $2.279(2)$ \\
\hline $\mathrm{Fa}_{4}$ & $\cdots$ & $0.00000781(2)$ & $0.0295475(8)$ & $1.020(3)$ \\
\hline$F a_{5}$ & $\cdots$ & $\cdots$ & $-0.0065382(8)$ & $0.587(3)$ \\
\hline$F a_{6}$. & $\cdots$ & $\cdots$ & $0.0015452(8)$ & $0.355(2)$ \\
\hline $\mathrm{Fa}_{7}$ & $\cdots$ & $\cdots$ & $-0.0003809(8)$ & $0.225(2)$ \\
\hline$F a_{8}$ & $\cdots_{i=1}$ & $\cdots$ & $0.0000943(8)$ & $0.147(2)$ \\
\hline$F a_{9}$ & $\cdots$ & $\cdots$ & $-0.000025(1)$ & $0.098(2)$ \\
\hline$F a_{10}$ & $\cdots$ & $\cdots$ & $\cdots$ & $0.070(2)$ \\
\hline$F a_{11}$ & $\cdots$ & $\cdots$ & $\cdots$ & $0.051(2)$ \\
\hline$F a_{12}$ & $\cdots$ & $\cdots$ & $\cdots$ & $0.040(2)$ \\
\hline$F a_{13}$ & $\cdots$ & $\cdots$ & $\cdots$ & ${ }^{\prime} 0.024(2)$ \\
\hline$F a_{14}$ & $\ldots$ & $\cdots \quad=-$ & $\cdots$ & $0.017(2)$ \\
\hline$\sigma$ & $2.2 \times 10^{-8}$ & $1.4 \times 10^{-7}$ & $4.2 \times 10^{-6}$ & 0.0089 \\
\hline
\end{tabular}

${ }^{2}$ Uncertainties are one standard deviation in the last digit. 
TABLE III. Point group symmetry operations $s^{\mathrm{a}}$ for $A(\sigma=0)$ and $E(\sigma= \pm 1)$ RESs obtained from the model torsion-rotation Hamiltonian in Eq. (5).

\begin{tabular}{lrrrc}
\hline \hline$C_{2 h}{ }^{\mathrm{b}}$ & \multicolumn{1}{c}{$A$ Species $(\sigma=0)^{\mathrm{c}}$} & $C_{s}{ }^{\mathrm{d}}$ & $E$ species $(\sigma= \pm 1)^{\mathrm{e}}$ \\
\hline$E$ & $J, \theta, \chi$ & $J_{x}, J_{y}, J_{z}$ & $J_{x}, J_{y}, J_{z}$ \\
$\sigma_{x z}$ & $J, \theta,-\chi$ & $+J_{x},-J_{y},+J_{z}$ & $\sigma_{x z}$ & $+J_{x},-J_{y},+J_{z}$ \\
$C_{2 y}$ & $J, \pi-\theta, \pi-\chi$ & $-J_{x},+J_{y},-J_{z}$ & $J, \theta,-\chi$ & \\
$i$ & $J, \pi-\theta, \pi+\chi$ & $-J_{x},-J_{y},-J_{z}$ & & \\
\hline
\end{tabular}

${ }^{2}$ The $J, \theta, \chi$ transformations can be used directly in Eq. (5). The $J_{x}, J_{y}, J_{z}$ transformations can be used in Eq. (5) after substituting from Eq. (1) to obtain an effective classical totational Hamiltonian of the form $H\left(J_{x}, J_{y}, J_{z}\right)$, but they cannot be used for the quantum mechanical operators $J_{x}, J_{y}, J_{z}$ in Eq. (2).

${ }^{b}$ This group is labeled $C_{2 h}$ here because the angular momientum components $J_{x}, J_{y}, J_{z}$, which function as Cartesian coordinates when drawing the RES, transform as expected for Cartesian coordinates under $C_{2 h}$.

" $A$ and $E$ refer to symmetry species of the torsion-rotation energy levels under symmetry operations of the permutation-inversion group $G_{6}$ appropriate for acetaldehyde and similar molecules. $A$ and $E$ symmetry species correspond to setting $\sigma=0$ or \pm 1 , respectively, in Eq. (5).

${ }^{\mathrm{d}}$ This group is labeled $C_{s}$ here because the angular momentum components transform as expected for Cartesian coordinates under $C_{s}$.

(5), but that on the other hand, the RESs for $\sigma= \pm 1$ occur in pairs related by $\mathbf{J} \rightarrow-\mathbf{J}$ and $\sigma \rightarrow-\sigma$.]

The search for stationary points requires solving two coupled transcendental equations. Once a stationary point is found, the eigenvalues of the $2 \times 2$ hessian matrix indicate its character. When both eigenvalues are nonzero, the stationary points are called Morse critical points and correspond to maxima (two negative eigenvalues), 'minima (two positive eigenvalues), or saddles (one positive, one negative eigenval-

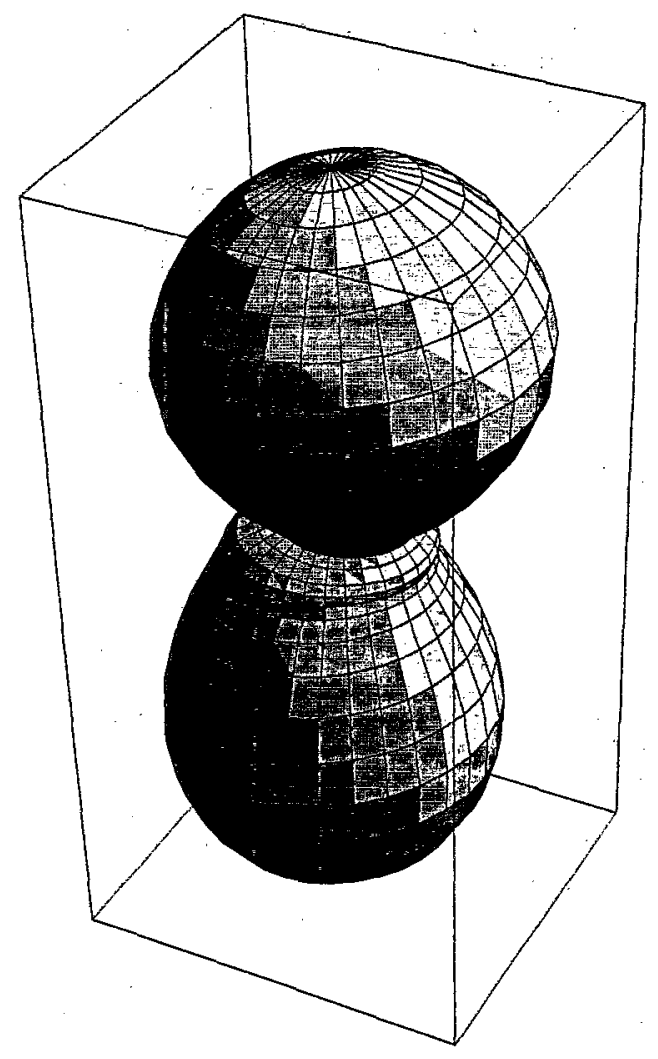

FIG. 1. The $J=10$ rotational energy surface for the $E$ species tunneling component of the $v_{t}=2$ torsional state of acetaldehyde, calculated from Eq. (5) using the constants in Tables I and II (with $F a_{0}=0$ ) and a quantum mechanical $J$ value of $\sqrt{ } 110$. The $z$ axis $(\theta=0)$ points vertically upward. This RES is essentially a somewhat distorted version of the RES for a nearprolate rigid asymmetric top with the rotational constants of acetaldehyde.

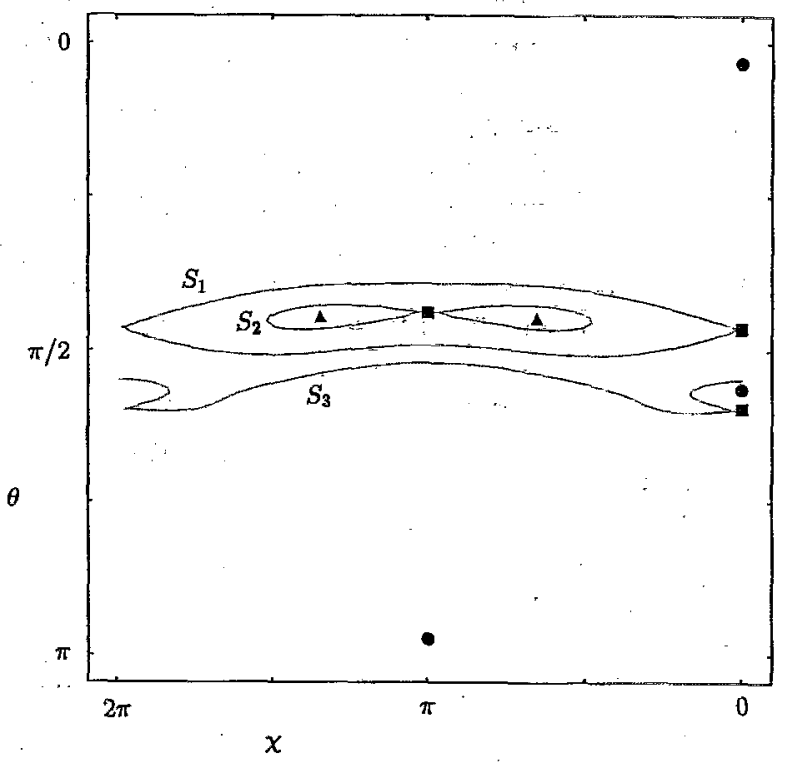

FIG. 2. Contour plot of energies for the $J=10, v_{t}=2, E$-species acetaldehyde rotational energy surface shown in Fig. 1 as a function of $0 \leqslant \chi \leqslant 2 \pi$ (abscissa) and $0 \leqslant \theta \leqslant \pi$ (ordinate). Stationary points on the RES are indicated by circles (maxima), squares (saddles), and triangles (minima). Contour lines indicate the three separatrices $\left(S_{1}, S_{2}, S_{3}\right)$, which are constant energy lines passing through the saddle points. This contour is viewed such that the positive $z$ axis points upward in the plane of the paper, and the positive $x$ and $y$ axes point upward from the plane of the paper at the points $(\theta, \chi)=(\pi / 2, \pi)$ and $(\pi / 2, \pi / 2)$, respectively. [Note that Eqs. (1) require that $\chi$ increase in a clockwise direction when viewed from the positive $z$ axis.] A vertical line at $\chi=0$ would correspond to the intersection of the $x z$ plane and the RES for negative $x$ values. This same intersection would reappear because of the periodicity in $\chi$ as a vertical line at $\chi=2 \pi$, but stationary points are not repeated there to avoid confusion in counting them. A vertical line at $\chi=\pi$ would correspond to intersection of the $x z$ plane and the RES for positive $x$ values. Vertical lines at $\chi=\pi / 2$ and $3 \pi / 2$ would correspond to intersections of the $y z$ plane and the RES for positive and negative $y$ values, respectively. A horizontal line at $\theta=\pi / 2$ would correspond to intersection of the $x y$ "equatorial" plane and the RES. The variation in "width" of the RES in Fig. 1 as a function of $\theta$ is not evident in this $(\theta, \chi)$ contour plot of the energies. An extreme example of this problem would be illustrated by horizontal lines at $\theta=0$ and $\pi$ in Fig. 2, each of which corresponds to a single point in Fig. I. As expected, the distribution of stationary points in this figure preserves the $C_{s}$ symmetry (reflection in the $x z$ plane) of the RES. 


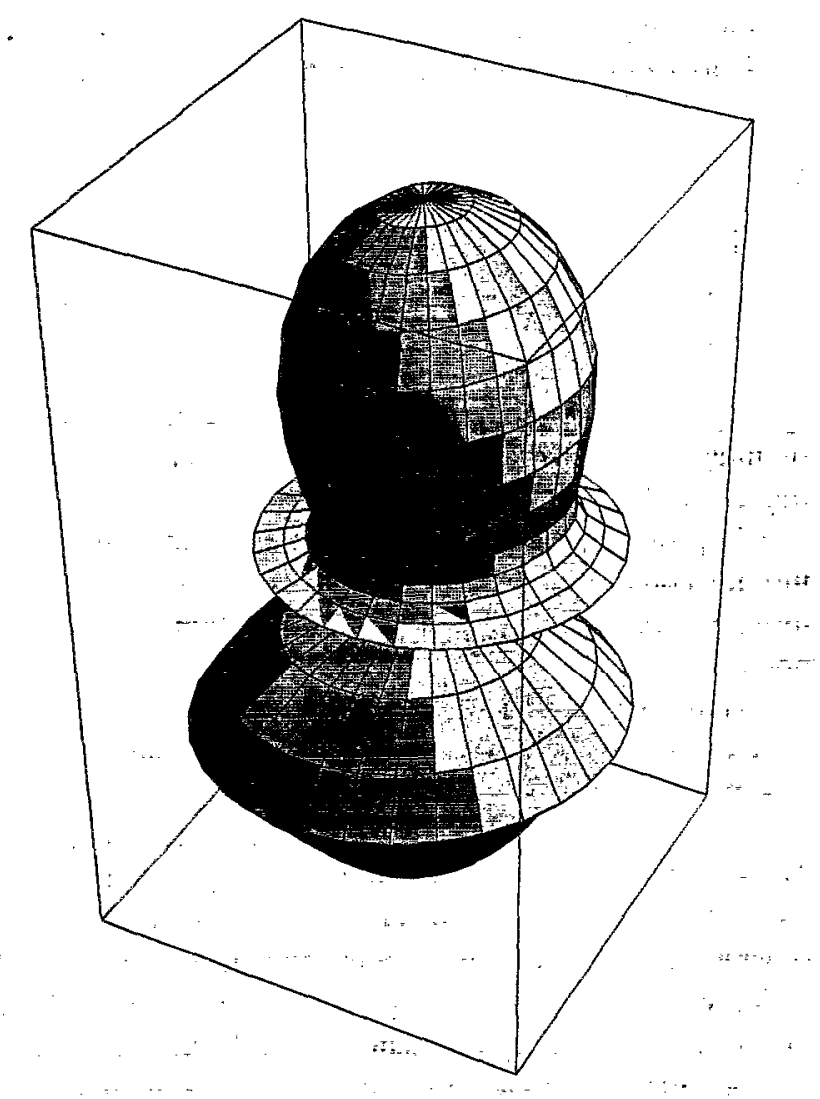

FIG. 3. The $J=10$ rotational energy surface for the $E$-species tunneling component of the $v_{i}=3$ torsional state of acetaldehyde, calculated from Eq, (5) using the constants in Tables $I$ and $\Pi$ (with $F a_{0}=0$ ) and a quantum mechanical $J$ value of $\sqrt{ } 110$. The $z$ axis $(\theta=0)$ points vertically upward. This RES deviates considerably from the RES for a near-prolate rigid asymmetric top with the rotational constants of acetaldehyde.

ue). When "one or both eigenvalues are zero, the stationary points are called non-Morse critical points. These points are also called bifurcation points because for them the stationary-point structure of the surface undergoes a change.

For comparison later, we recall ${ }^{1}$ that six stationary points are found for the rigid asymmetric rotor: two maxima on the $a$ axis, two minima on the $c$ axis, and two saddles on the $b$ axis; and no bifurcations appear even when $J$ increases without limit.

The number of stationary points found for our model Hamiltonian for $v_{t}=0,1$, and $2 \mathrm{~A}$ states is six, just as for the rigid asymmetric rotor RES. The two minima are located on the RAM $y$ axis (i.e., on the PAM $c$ axis) at $(\theta, \chi)=(\pi / 2, \pi / 2)$, $(\pi / 2,3 \pi / 2)$. The two maxima and the two saddles lie in the RAM $x z$ plane (the PAM $a b$ plane). However, the two maxima lie neither exactly on the RAM $z$ axis nor exactly on the PAM $a$ axis; though for $v_{t}=0,1$, and 2 in $\mathrm{CH}_{3} \mathrm{CHO}$, they lie closer to the PAM $a$ axis. Furthermore, the axis passing through the saddles is not orthogonal to the axis.passing through the maxima, though it lies close to the PAM $b$. axis. This picture is valid for $v_{t}=0,1$, and 2 torsional states of $A$ symmetry for all $J$ values examined.

For $v_{t}=0$ and $1 E$-state RESs, the number of stationary points is still six, and axes passing through the maxima,

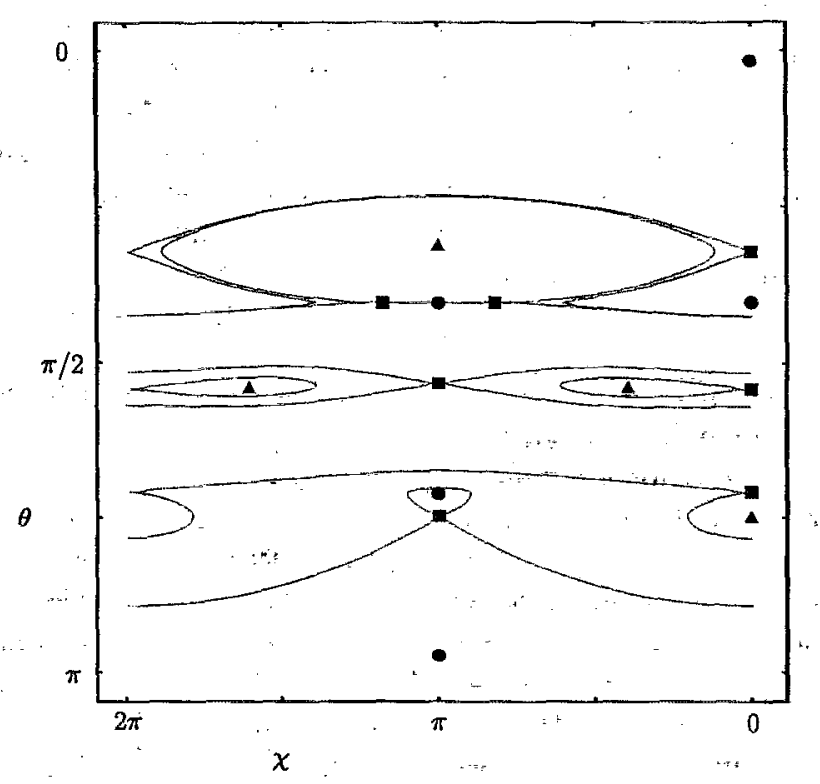

FIG. 4. Contour plot of energies for the $J=10, v_{t}=3, E$-species acetaldehyde rotational energy surface shown in Fig. 3 as a function of $0 \leqslant \chi \leqslant 2 \pi$ and $0 \leqslant \theta \leqslant \pi$. Stationary points on the RES are again indicated by circles (maxima), squares (saddles), and triangles (minima); contour lines passing through the saddles again indicate the separatrices. (See the caption to Fig. 2 for further details.) Note that the separatrices passing above and below the maximum near $(\theta, \chi)=(1.27, \pi)$ are not resolved in this figure and appear as a single straight line.

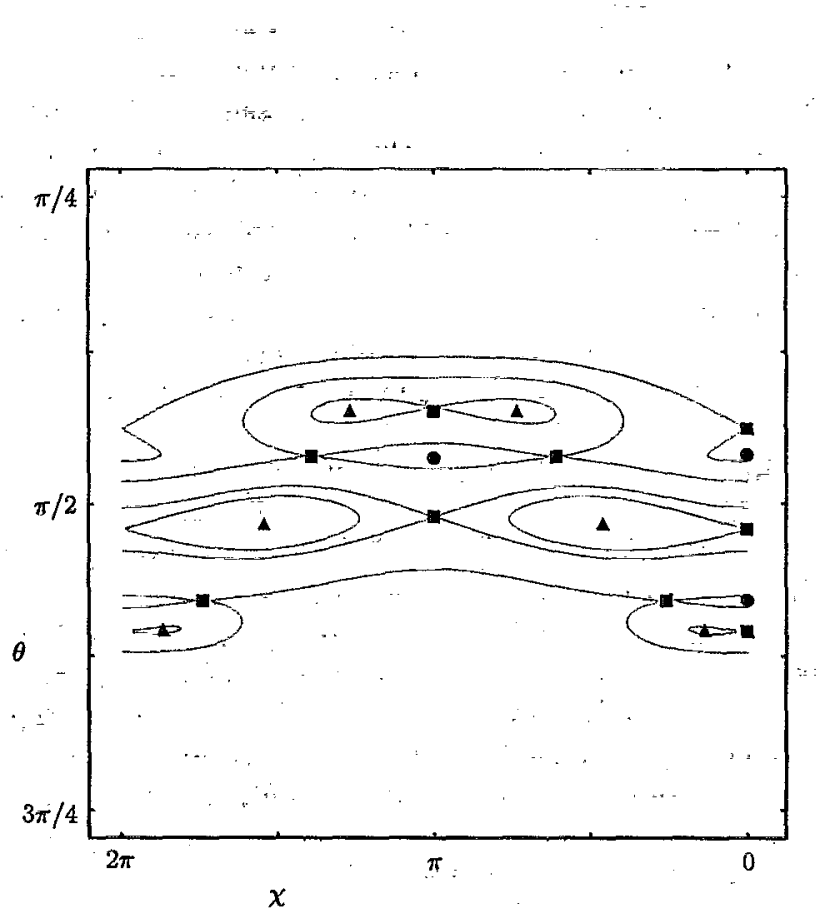

FIG. 5. Contour plot of energies for the $J=25, v_{t}=3, E$-species acetaldehyde rotational energy surtace in the region $0 \leqslant \chi \leqslant 2 \pi$ and $\pi / 4 \leqslant \theta \leqslant 3 \pi / 4$. Maxima, saddles, and minima in this region are indicated by circles, squares, and triangles, respectively. Contour lines indicate the separatrices. (See the caption to Fig. 2 for further details). Many of the nine maxima and minima in this figure do not support quantizing trajectories and thus do not have simple quantum mechanical significance. 
saddles, and minima are still close to the $a, b$, and $c$ principal axes, respectively. The axes passing through the two maxima and the two saddles still lie in the RAM $x z$ (PAM $a b)$ plane, but these four axes no longer coincide in pairs (i.e., there is no axis containing both maxima and no axis containing both saddles). The axes passing through the two minima no longer lie on the $y$ axis and no longer exactly coincide, though they are related by reflection in the $x z$ plane.

In spite of the qualitative differences described in the preceding two paragraphs, RESs for $v_{t}=0,1$, and $2 A$ and $v_{t}=0,1 E$ torsional tunneling components of acetaldehyde are still rather similar to RESs for a rigid near prolate asymmetric top in the sense that all of these RESs have stationary points corresponding to two maxima $(M)$, two saddles $(s)$, and two minima $(m)$ lying on, or nearly on, the principal inertia axes of the molecule. For higher $v_{t}$ states, new characteristics emerge. Thus, for the $E$ state RESs with $v_{t}=2$ and $J=1$, there are only two stationary points $(M, m)$; for $2 \leqslant J \leqslant 4$, there are four $(2 M, s, m)$; for $5 \leqslant J \leqslant 7$, there are six $(2 M, 2 s, 2 m)$, as for the rigid asymmetric rotor; for $8 \leqslant J \leqslant 30$, there are eight $(3 M, 3 s, 2 m)$; and for $J \geqslant 31$, there are again six stationary points $(2 M, 2 s, 2 m)$. Evidently, a number of catastrophes occur for $v_{t}=2 E$-state RESs which create or annihilate $(M, s)$ or $(m, s)$ pairs of stationary points. Classically, all new maxima and minima give rise to stable rotation axes. Quantum-mechanical implications of these new stationary points are discussed in the next section.

Figure 1 shows an $E$-species rotational energy surface for $v_{t}=2, J=10$, which has been altered by removing the constant $F a_{0}$ term of the energy function in order to show more clearly the strong deformation in the equatorial region. Figure 2 shows a contour plot of this same surface, which displays the positions of the stationary points more clearly. The separatrices (contour lines passing through the saddle points) are also shown. A comparison of the topology of this surface with that for a rigid asymmetric top shows that the maximum and saddle near $\theta=1.77$ and $1.87 \mathrm{rad}$, respectively, are new. Furthermore, the separatrix structure is considerably more complicated (there are now three separatrices instead of one).

In Fig. 3, the $E$-species rotational energy surface for $v_{t}=3, J=10$ is shown. As can be seen from the corresponding contour plot in Fig. 4, the number of stationary points $(5 M, 7 s, 4 m)$ has increased dramatically with respect to the $v_{t}=0,1$, or 2 RESs. The contour plot for $J=25$ in Fig. 5 shows 18 of the 20 stationary points $(5 M, 9 s, 6 \mathrm{~m})$ and a large number of separatrices. Just as for $v_{t}=2$, the complicated creation and annihilation history (as $J$ increases) of the various stationary points for $v_{t}=3$ could be chronicled in terms of catastrophes, beginning with the stationary points $(2 M, 1 \mathrm{~s}, 1 \mathrm{~m})$ which exist for $J=1$.

\section{Quantizing trajectories}

A knowledge of the RES topology is convenient when searching for trajectories which quantize the action. ${ }^{16}$ In principle, any axis can be used to write the one-dimensional semiclassical quantization condition

$$
\int_{0}^{2 \pi} J_{\gamma} d \gamma=(n+\alpha / 4) h
$$

where $n$ is an integer, $h$ is Planck's constant, $\gamma$ is an angle running from 0 to $2 \pi$ which describes motion around the trajectory, $J_{\gamma}$ is the momentum conjugate to this angle, and $\alpha$ is the Maslov index, ${ }^{19}$ which takes into account the effects of turning points during the trajectory: For rotations (with no turning points), $\alpha=0$; for vibrations (with two turning points), $\alpha=2$.

Traditionally, one first seeks the quantizing trajectories which surround classically stable rotation axes (i.e., axes passing through maxima and minima on the RES), beginning with trajectories as close to the stationary point as possible. The search for trajectories around a given axis ends when the energy of the trajectory reaches the energy of the nearest separatrix. From this point of view, the classical catastrophes which create new stationary points, as described in the previous section, will have quantum mechanical significance only to the extent that the new stationary points support quantizing trajectories.

The search for trajectories around a given stationary point is sometimes simplified by a transformation of the original Hamiltonian $H_{\mathrm{RAM}}$ given in Eq. (5) to a Hamiltonian written in a new axis system with the new $z$ axis passing through the stationary point. If $\left(J, \theta_{c}, \chi_{c}\right)$ are the spherical polar coordinates of this stationary point in the RAM system, then the angular momentum components given by Eqs. (1) in the RAM axis system can be related to those in the new axis system in the following way:

$$
\begin{aligned}
\left(\begin{array}{l}
J_{x} \\
J_{y} \\
J_{z}
\end{array}\right)_{\mathrm{RAM}}= & \left(\begin{array}{ccc}
\cos \chi_{c} & \sin \chi_{c} & 0 \\
-\sin \chi_{c} & \cos \chi_{c} & 0 \\
0 & 0 & 1
\end{array}\right) \\
& \times\left(\begin{array}{ccc}
\cos \theta_{c} & 0 & -\sin \theta_{c} \\
0 & 1 & 0 \\
\sin \theta_{c} & 0 & \cos \theta_{c}
\end{array}\right)\left(\begin{array}{l}
J_{x} \\
J_{y} \\
J_{z}
\end{array}\right)_{\text {new }} .
\end{aligned}
$$

The RAM momenta expressed in terms of the new momenta by Eq. (10) can be substituted in Eq. (5) after converting it back from a function of $(J, \theta, \chi)$ to a function of $\left(J_{x}, J_{y}, J_{z}\right)_{\mathrm{RAM}}$ using Eqs. (1). $\left(J_{x}, J_{y}, J_{z}\right)_{\text {new }}$ in this RES can then be expressed in terms of coordinates $(J, \beta, \gamma)$ defined by equations analogous to Eqs. (1). Alternatively, $\left(J_{x}, J_{y}, J_{z}\right)_{\text {new }}$ can be expressed in terms of $\left(J, J_{\gamma}, \gamma\right)$, where $J_{\gamma}=J \cos \beta$. The rotational energy surface of Eq. (5) in the rotated axis system expressed in these latter coordinates takes the form 


$$
\begin{aligned}
E\left(J_{\gamma}, \gamma\right)= & \sum_{n} F a_{n} \cos (2 n \pi / 3)\left\{\rho\left[\cos \theta_{c} J_{\gamma}-\sin \theta_{c}\left(J^{2}-J_{\gamma}^{2}\right)^{(1 / 2)} \cos \gamma\right]-\sigma\right\}+A^{\prime \prime} J_{\gamma}^{2}+B^{\prime \prime}\left(J^{2}-J_{\gamma}^{2}\right) \cos ^{2} \gamma \\
& +C^{\prime \prime}\left(J^{2}-J_{\gamma}^{2}\right) \sin ^{2} \gamma-2 D_{x y}^{\prime \prime}\left(J^{2}-J_{\gamma}^{2}\right) \sin \gamma \cos \gamma+2 D_{y z}^{\prime \prime} J_{\gamma}\left(J^{2}-J_{\gamma}^{2}\right)^{(1 / 2)} \sin \gamma-2 D_{x z}^{\prime \prime} J_{\gamma}\left(J^{2}-J_{\gamma}^{2}\right)^{(1 / 2)} \cos \gamma
\end{aligned}
$$

where the constants $A^{\prime \prime}, B^{\prime \prime}, C^{\prime \prime}, D_{x y}^{\prime \prime}, D_{y z}^{\prime \prime}$, and $D_{x z}^{\prime \prime}$, defined by analogy with the constants in the quantum mechanical Hamiltonian of Eq. (4), are functions of the constants $A, B$, $C$, and $D_{x z}$ of $H_{\mathrm{RAM}}$ in Eq. (4), and of the coordinates $\theta_{c}, \chi_{c}$ of the stationary point to which the $z$ axis has been rotated. The function $J_{\gamma}(E, \gamma)$ cannot be explicitly obtained, so Eq. (11) must be solved numerically when performing the integrations in Eq. (9).

We now apply the quantization condition of Eq. (9) to the $v_{t}=2, J=10, E$-species RES shown in Figs. 1 and 2, since the results obtained for this example illustrate a number of general points. The maximum near $(\theta, \chi)=(0.10,0)$ in Fig. 2 lies approximately on the positive side of the principal $a$ axis of the acetaldehyde molecule. This maximum supports eight quantizing rotational trajectories with $10 \geqslant n \geqslant 3$ in Eq. (9). Similarly, the maximum near $(3.07, \pi)$ lies approximately on the negative side of the $a$ axis; it supports nine rotational trajectories with $10 \geqslant-n \geqslant 2$. Note that because the $C_{s}$ symmetry of $E$-state RESs for acetaldehyde is considerably lower than the $D_{2 h}$ symmetry of RESs for rigid asymmetric rotors, trajectories around the positive and negative $a$ axes are not related to each other by symmetry and in particular are not equal in number.

The regions between the two minima and their nearest separatrix $\left(S_{2}\right)$, or between the maximum near $(1.77,0)$ and its nearest separatrix $\left(S_{3}\right)$, turn out to be too small to support any quantizing trajectories for $J=10$.

Consider next trajectories in regions bounded by two separatrices. The region between $S_{1}$ and $S_{3}$ is relatively simple. It supports two rotational trajectories around the $a$ axis, with $n=-1$ and 0 . The region between $S_{1}$ and $S_{2}$ is more complicated. Inspection of Fig. 2 shows that no rotational trajectories around the $a$ axis are possible. Rotational trajectories around the saddle near $(1.38, \pi)$ are possible, and we initially moved the $z$ axis to this saddle and then tried to. use the resulting trajectories around this new $z$ axis in Eq. (9). These attempts were not successful because such trajectories led to triple-valued functions for $\beta(\gamma)$, and consequently also for $J_{\gamma}(\gamma)$, which could not be handled by our numerical computational procedures. We therefore returned the $z$ axis to the maximum near $(0.10,0)$ and treated the trajectories in the region between $S_{1}$ and $S_{2}$ as vibrations in Eq. (9). The functions $\beta(\gamma)$ were still double valued, but they proved to be much simpler to handle in our numerical procedures, so that three quantizing vibrational trajectories with respect to the $a$ axis were found between $S_{1}$ and $S_{2}$, with $n=2,1$, and 0 .

In summary, all of the $2 J+2=22$ trajectories expected for a primitive semiclassical quantization of an asymmetric rotor were found. Of these, 17 correspond closely to the rotational trajectories around the $a$ axis obtained for the simple asymmetric-top RES of an acetaldehyde molecule in the ab- sence of internal rotation. The two trajectories lying between separatrices $S_{1}$ and $S_{3}$ are also rotational trajectories around the $a$ axis, and in that sense, they are topologically similar to the corresponding trajectories on the simple asymmetric top RES. The three trajectories lying between separatrices $S_{1}$ and $S_{2}$, however, which would be rotational trajectories on the simple asymmetric top RES, have become vibrational trajectories for $J=10$ in the $v_{t}=2 E$ torsional state of acetaldehyde. Thus, as expected, the classical catastrophes giving rise to altered stationary-point and separatrix structure lead to qualitative differences in trajectory structure.

An examination of RES topology is frequently useful for predicting the occurrence of rotational energy level clusters. ${ }^{1,3-5}$ Such clustering occurs when (i) a number of symmetrically equivalent stationary points on the RES give rise to the same number of symmetrically equivalent classical trajectories; and (ii) energy shifts induced by quantum mechanical tunneling effects among the semiclassically quantized equivalent trajectories are small. For high $J$ and low $v_{t}$, acetaldehyde RESs (and therefore acetaldehyde rotational energy level clusters) are expected to be similar to those for a simple asymmetric rotor without internal rotation. To search for clusters at lower $J$ and higher $v_{t}$, it is necessary to examine contour plots like those in Figs. 2, 4 and 5. For example, the two minima in Fig. 2 are related by reflection in the $x z$ plane of symmetry, so that trajectories around these minima should come in degenerate pairs (twofold clusters). Even though the regions between these minima and their nearest separatrices are too small to support quantizing trajectories for $J=10$, quantizing trajectories should become possible at higher $J$. It would be interesting to search experimentally for the onset of such oblate-type clusters.

\section{APPLICATION-LABELING OF $v_{t}=2, J=10, E$ SYMMETRY STATES}

As a final topic, we examine the quantum mechanical implications of the use of various axes in the action integral of Eq. (9). Constant energy trajectories very close to a maximum or minimum stationary point take the form of an ellipse whose plane is perpendicular to the stable axis passing through the stationary point. If this axis is used to define $\gamma$ and $J_{\gamma}$, then the integrand $J_{\gamma}(E, \gamma)$ in Eq. (9) is nearly constant with respect to the angle $\gamma$ for a given trajectory.

On the other hand, trajectories near separatrices, or trajectories which surround several stationary points, do not have such simple forms, and $J_{\gamma}$ in general varies greatly along the trajectory. The question then arises of which axis leads to the minimum variation in $J_{\gamma}$. This question has quantum mechanical significance because a quantum calculation using a basis set with the angular momentum projection quantum number $K$ quantized along this axis will lead to 
eigenfunctions with the least mixing of basis functions with different $K$ values, i.e., to eigenfunctions in which $K$ is as good a quantum number as possible. It seems reasonable to suppose that from this point of view, the best axis of quantization for an arbitrary trajectory lies along the direction of the time average of the angular momentum of the trajectory $\overline{\mathbf{J}}_{t}$, where (for rotational trajectories)

$$
\overline{\mathbf{J}}_{t}=\int_{0}^{2 \pi} \mathbf{J}(E, \gamma) \dot{\gamma}^{-1} d \gamma / \int_{0}^{2 \pi} \dot{\gamma}^{-1} d \gamma .
$$

The three components of $\mathbf{J}(E, \gamma)$ are given by equations analogous to Eqs. (1): Such a definition of best axis of quantization implies the use of different axes of quantization for trajectories in different regions. It can be shown that when the desire for minimum variation in $J_{\gamma}$ is applied to the difficult region between the separatrices $S_{1}$ and $S_{2}$ in Fig. 2, it clearly favors use of an axis through the maximum near the principal $a$ axis together with vibrational trajectories, rather than use of an axis through the saddle near the principal $b$ axis together with rotational trajectories.

Other criteria for choosing the best quantization axis are possible, however, depending on the purpose for which the axis is to be used. At least three candidates for best axis suggest themselves for a near prolate rotor with a symmetrictop internal rotor: (i) the principal axis of smallest moment of inertia; (ii) the symmetry axis of the internal rotor; or (iii) the direction of the vector $\rho$ appearing in Eq. (3). The Hamiltonian most often used for quantum mechanical calculations of torsion-rotation energy levels is $H_{\text {RAM }}$ described above, because it can be diagonalized easily in a two-step procedure. Kleiner et al. ${ }^{12}$ have reported finding ambiguities in the labeling of many states obtained from $H_{\mathrm{RAM}}$ during the process of analyzing the spectra of acetaldehyde, because for many cases, several basis functions contribute with approximately the same weight to a given eigenvector. This problem complicates the assignment procedure. Although a scheme has been devised ${ }^{20}$ to analyze unassignable spectra based on the use of energy ordering to label states, it is not appropriate to apply such a method to the acetaldehyde spectrum for several reasons. First of all, the spectrum is not congested enough to prevent assignment. Second and more important, the spectrum is fit to a phenomenological Hamiltonian using an iterative procedure. When the parameters change from one cycle to the next, the energy ordering can easily change in such a way that in different cycles, different assignments would be used. Kleiner and co-workers managed to solve their problems using the essentially experimental criterion of requiring smooth $B$ values for calculated series of energy levels of given $K$ and increasing $J$.

The problems encountered when using the RAM $z$ axis for $K$ labeling purposes (as Kleiner and co-workers did) is illustrated graphically by the dashed lines in Fig. 6,- which connect values of the squares of coefficients of RAM basis set functions of various $K$ for three selected eigenvectors of $E$ symmetry characterized by $v_{t}=2$ and $J=10$. Based on these RAM coefficients, the three energy levels should be called $\left|v_{t} ; J, K\right\rangle=|2 ; 10,3\rangle,|2 ; 10,4\rangle$, and $|2 ; 10,3\rangle$. This result is clearly awkward, since there are two eigenfunctions labeled by exactly the same quantum numbers.
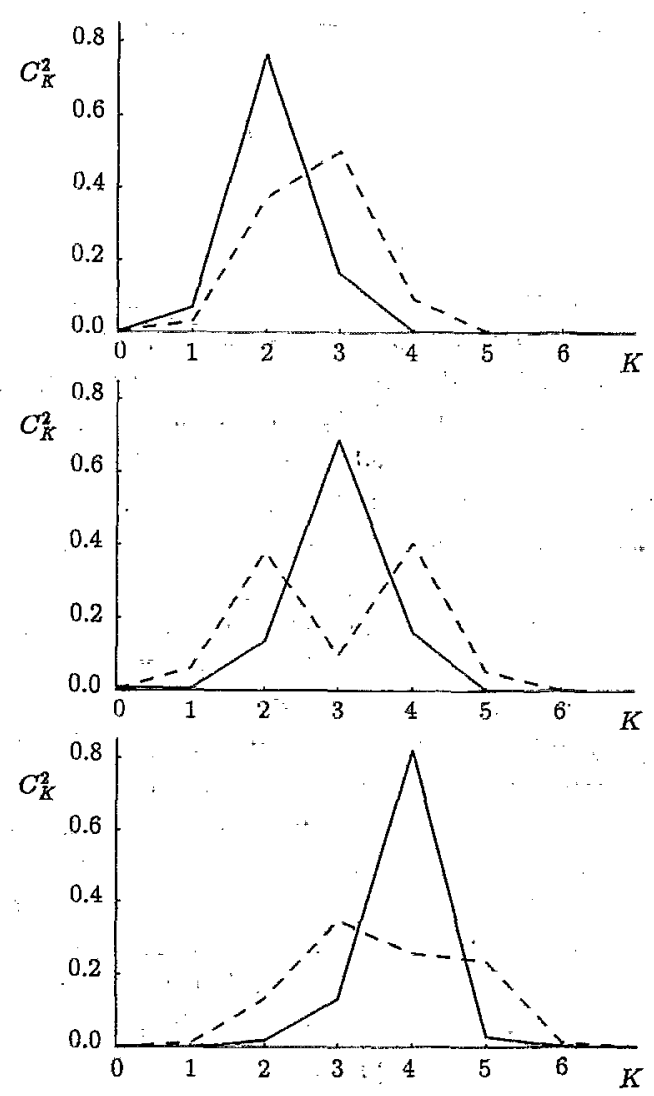

FIG. 6. Squares of the coefficients $\left(C_{K}^{2}\right)$ of basis functions of given $K$ for three selected $v_{t}=2$ eigenvectors of acetaldehyde with $E$ torsional symmetry and $J=10$. Dashed lines connect coefficients in the rho-axis-method basis set $\left(H_{\text {RAM }}\right)$ used for actual calculations in Ref. 12. These coefficients lead to the awkward $K$ labels of 3, 4, 3 for these three eigenyectors.-Solid lines connect coefficients of the same eigenvectors using basis functions referred to an axis system whose $z$ axis passes through the appropriate maximum of the rotational energy surface. These coefficients lead to the reasonable $K$ labels of $2,3,4$ for these three eigenvectors.

This ambiguity can be removed completely by making a rotation of the eigenvectors to the RES axis system, which for $v_{t}=2$ of acetaldehyde is almost coincident with the PAM axis system. Suich a rotation is easily accomplished by using the Wigner $D$ matrices. Coefficients based on this axis system (connected by solid lines in Fig. 6) indicate that these same three energy levels should be called $\left|v_{t} ; J, K\right\rangle=|2 ; 10,2\rangle,|2 ; 10,3\rangle$, and $|2 ; 10,4\rangle$; so that all ambiguities have disappeared.

\section{CONCLUSION}

The present work represents the first step in the description of rotational energy. surfaces of acetaldehyde-like molecules for torsion-rotation levels below the barrier to internal rotation. It was hoped at the start of this work that the classical description of various quantizing trajectories on the RES would be more enlightening than the traditional quantum mechanical eigenvectors: expressed in the free-rotor times overall-rotation product basis set. 
On the one hand, however, the large number of catastrophes which occur as $J$ varies (together with changes in separatrix structure, changes in trajectories from rotational to vibrational, etc.) suggest that a simple general classical treatment of the RESs for all $J$ (even with given $v_{t}$ and $A, E$ symmetry species) will be difficult, and that it may be necessary to treat the RESs for many $J$ values as special cases.

On the other hand, the $\Delta J=1$ rotational energy intervals ( $B$ values) calculated quantum mechanically vary smoothly with $J$, suggesting that "catastrophic" effects on the rotational energy structure may be absent. Their effect on other spectroscopic observables in the internal rotor problem has not been investigated, however, and the classical catastrophes found in the rotational energy surfaces could be associated with a breakdown of the $K$ quantum number, giving rise at the quantum level to changes in the intensity of particular lines, or to the presence of forbidden transitions. It is easy, e.g., to imagine cases where fairly pure $K$ projections (for labeling purposes) can only be obtained by using axes passing through different maxima on the rotational energy surface, so that the quantum mechanical selection rules written in terms of these different $K$ 's would have little validity.

To obtain more information on this question, we will continue this work in two ways. First, we plan to investigate in detail the relation between changes in classical trajectories and changes in quantum mechanical eigenvalues and eigenvectors for $v_{t}=2$ in acetaldehyde, i.e., for the last torsional level below the top of the barrier. Second, we plan to investigate torsional levels above the barrier, although for such levels, an accurate method for treating quantum tunneling and interference effects between neighboring or close-lying trajectories on intersecting RESs is essential if the classical description is to be truly useful, and not just interesting, for the internal rotation problem in acetaldehyde.

\section{ACKNOWLEDGMENTS}

This work was supported in part by the Division of Chemical Sciences, Office of Basic Energy Sciences, Office of Energy Résearch, U.S. Department of Energy, and by the Consejo Superior de Investigaciones Científicas of Spain. The authors are indebted to Dr. D. A. Sadovskií and Dr. I. M. Pavlichenkov for helpful criticism of the manuscript.
'W. G. Harter and C. W. Patterson, J. Chem. Phys. 80, 4241 (1984).

${ }^{2}$ W. G. Harter, Comput. Phys. Rep. 8, 319 (1988).

${ }^{3}$ J. Makarewicz, Mol. Phys. 69, 903 (1990).

${ }^{4}$ B. I. Zhilinskií and I. M. Pavlichenkov, Opt. Spektrosk. 64, 688 (1988); J. Makarewicz and J. Pyka, Mol. Phys. 68, 107 (1989).

${ }^{5}$ I. N. Kozin, S. P. Belov, O. L. Polyansky, and M. Yu. Tretyakov, J. Mol. Spectrosc. 152, 13 (1992); M. Yu. Tretyakov, S. P. Belov, I. N. Kozin, and O. L. Polyansky, ibid. 154, 163 (1992); I. N. Kozin, O. L. Polyansky, S. I. Pripolzin, and V. L. Vaks, ibid. 156, 504 (1992); I. N. Kozin, S. Klee, P. Jensen, O. L. Polyansky, and I. M. Pavlichenkov, ibid. 158, 409 (1993).

${ }^{6}$ I. M. Pavlichenkov and B. I. Zhilinskií, Ann. Phys. (N.Y.) 184, 1 (1988); I. M. Pavlichenkov, Phys. Rep. 226, 173 (1993).

${ }^{7}$ R. Gilmore, Catastrophe Theory for Scientists and Engineers (Dover, New York, 1993).

${ }^{8}$ D. A. Sadovskií and B. I. Zhilinskií, Phys. Rev. A 47, 2653 (1993):

${ }^{9}$ V. B. Pavlov-Verevkin, D. A. Sadovskií, and B. I. Zhilinskií, Europhys. Lett. 6, 573 (1988); D. A. Sadovskií, B. I. Zhilinskií, J. P. Champion, and G. Pierre, J. Chem. Phys. 92, 1523 (1990); D. A. Sadovskií and B. I. Zhilinskií, Mol. Phys. 65, 109 (1988); V. M. Krivtsun, D. A. Sadovskii, and B. I. Zhilinskií, J. Mol. Spectrosc. 139, 126 (1990).

${ }^{10}$ L. Xiao and M. E. Kellman, J. Chem. Phys. 90, 6086 (1989); 93, 5805 (1990); Z. Li, L. Xìoo, and M. E. Kellman, ibid. 92, 2251 (1990).

${ }^{11}$ M. E. Kellman and L. Xiao, J. Chem. Phys. 93, 5821 (1990).

${ }^{12}$ S. P. Belov, M. Yu. Tretyakov, I. Kleiner, and J. T. Hougen, J. Mol. Spectrosc. 160, 61 (1993). This paper is the fifth in a series of papers on the analysis of the acetaldehyde microwave and far infrared spectra. For earlier papers dealing with smaller data sets, see I. Kleiner, M. Godefroid, M. Herman, and A. R. W. McKellar, J. MoI. Spectrosc. 142, 238 (1990); I. Kleiner, J. T. Hougen, R. D. Suenram, F. J. Lovas, and M. Godefroid, ibid: 148, 38 (1991); 153, 578 (1992); W. L. Barclay, Jr., M. A. Anderson, L. M. Ziurys, I. Kleiner, and J. T. Hougen, Astrophys. J. Suppl. 89, 221 (1993). Details concerning the model employed can be found in E. Herbst, J. K. Messer, F. C. De Licia, and P. Helminger, J. Möl. Spectrosc. 108, 42 (1984); K. V. L. N. Sastry, E. Herbst, R. A. Booker, and F. C. De Lucia, ibid. 116, 120 (1986).

${ }^{13}$ E. B. Wilson, J. C. Decius, and P. C. Cross, Molecular Vibrations (McGraw-Hill, New York, 1955).

${ }^{14}$ C. C. Lin and J. D. Swalen, Rev. Mod. Phys. 31, 841 (1959).

${ }^{15} \mathrm{~J}$. T. Hougen, I. Kleiner, and M. Godefroid, J. Mol. Spectrosc.: 163, 559 (1994).

${ }^{16}$ C. W. Patterson, J. Chem. Phys. 83, 4618 (1985); see also D. Richards, J. Phys. B 16, 749 (1983) for an adiabatic rotational energy surface obtained by perturbation theory for a hydrogen atom in a magnetic field.

${ }^{17}$ R. T. Skodje, F. Borondo, and W. P. Reinhardt, J. Chem. Phys. 82, 4611 (1985); B. R. Johnson, ibid. 83, 1204 (1985).

${ }^{18}$ H. Leipholz, Stability Theory (Wiley, New York, 1987).

${ }^{19}$ I. C. Percival, Adv. Chem. Phys. 36, 1 (1977)

${ }^{20} \mathrm{~J}$. M. Standard, E. D. Lynch, and M. E. Kellman, J. Chem. Phys. 93, 159 (1990). 